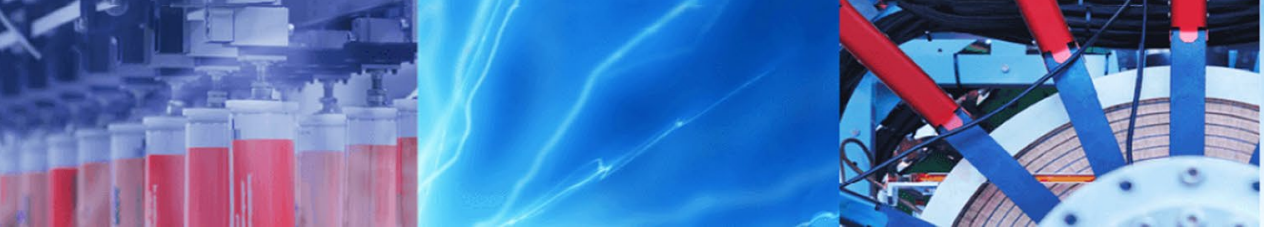

Research Article

\title{
An intelligent and personalized course advising model for higher educational institutes
}

\author{
Leul Aynekulu Tilahun ${ }^{1} \cdot$ Boran Sekeroglu²
}

Received: 10 March 2020 / Accepted: 26 August 2020 / Published online: 5 September 2020

(c) Springer Nature Switzerland AG 2020

\begin{abstract}
In this study, we present an intelligent personalized course advising model by analyzing, selecting, and modifying effective course advising technologies. It overcomes the existing course advising systems by using common design for all Higher Educational Institutes and making the rule, regulations, curriculum, and grading system more flexible to change without affecting persistent academic data. The proposed model helps students by analyzing and suggesting appropriate courses according to their academic history and special interest via harmonized knowledge of different experts. To develop this model, analyze various requirements were gathered and explicitly analyzed from the course advisory systems and other related technologies, that were used in seven institutes. The proposed model contains seven components, in which they use association rule, rule-based expert system, and fundamental recommendation algorithms according to their suitability for collected requirements. We made more modifications and enhancements on those algorithms to achieve the purpose of each component. We used ISO-25010 quality model with the institutional rules and regulations of three departments to evaluate the proposed model. The obtained results showed that the proposed methodology of the model is an effective way to improve the efficiency of students course advisory systems. It also indicates that advisors and students can save more time through effective and efficient course suggestions during the course registration period.
\end{abstract}

Keywords Course advising $\cdot$ Intelligent systems $\cdot$ Expert system $\cdot$ Recommendation system

\section{Introduction}

Nowadays, countries invest in education to enhance knowledge, understanding, skills, values, and actions of the society. It is also a powerful tool for the development of social, cultural, economic, and political activities, which ensures environmental safety and conservation, promotes social fairness and encourages monetary sustainability.

Professional advice is required for students on different academic issues, especially during course registration periods. Sometimes, students are not able to make a proper decision on course registration, so those advisers are taking additional responsibilities to advise, motivate, and support each student as individuals.
Academic advising should be multi-dimensional, intentional, and grounded in the education system, which has to be included required materials to make specied outcomes for student learning. It has an essential importance in decision-making phases of unstructured and more complicated situations, which makes ambiguity to produce an exact response.

When students face problems or difficulties in course registration, they discuss the issues with their advisers, and an adviser advises by analyzing the students' situation according to his/her knowledge, skill, and experience to support students and to solve the issue. Academic advisory system is a set of activities for the dissemination of knowledge that guides students for the career goals and

Boran Sekeroglu, boran.sekeroglu@neu.edu.tr; Leul Aynekulu Tilahun, leul.aynekulu@wu.edu.et; leul.aynekulu@gmail.com |

${ }^{1}$ Kombolcha Institute of Technology, Wollo University, Wollo, Ethiopia. ${ }^{2}$ Near East University, Mersin 10, Nicosia, Cyprus, Turkey. 
development of academic plans [1]. The quality of academic advising has prime importance in improving students' academic performance. Effective academic advice creates desired results, while ineffective advice has a detrimental impact on the education system and wisdom development of students [2]. The ideal academic advising includes the training of students to know how to achieve their goals and provide a manageable and straightforward way to solve different academic, social, or personal problems that occurred in academic life. Therefore, academic advising becomes more difficult, complicated, and timeconsuming task, especially when the number of student are increased and adviser to student ratio reduced.

The emergences of computerized course advisory systems help to reduce the responsibility of academic advisers by assisting both the students and academic advisers. Nevertheless, the problem-solving mechanisms are varied from one system to another one, which was designed under strong consideration of user requirements of a particular Higher Education Institutes (HEI).

Various problems and gaps are noted for existing general systems. Each student has varied academic records; thus, the analysis process is a complicated task both for academic advisers and computerized systems to provide accurate course suggestions for the current academic semester. Academic rules and regulations have differences for regular and irregular students or freshmen and graduating students that cause mistakes in the application of rules and regulations properly to each student. But, one of the most critical gaps of existing systems is the consideration of all available courses without considering students' interests or success in related courses, particularly in elective courses. For this reason, several studies were performed in electronic advising systems [3-8]

Dash and Vaidhehi [9] developed an advising system for the students of the eighth standard of Indian Certificate of Secondary Education (ICSE) board to choose their electives using machine-learning algorithms to assist human advisers efficiently. It assisted Indian school students in analyzing their academic history and helped them to choose their electives wisely.

Huang et al. [10] developed a course recommendation system for Massive Open Online Courses (MOOCs) platform that contributes to an improvement of course recommendation model and algorithm based on distributed computational framework. Multivariate Curve Resolution (MCR) used distributed association rule mining algorithms to improve priori algorithm that is useful to mine the hidden course rule in course registration. In another study, Hadoop and Spark concluded that the MCRs is the most efficient algorithm for course registration systems. Those researchers have modeled and tested Naive Bayes, J48, Projective Adaptive Resonance Theory (PART), Random
Forest, and Instant Based Learner (IBK) on dierent data set. As a result, the accuracy of the classification models, namely, random forest, PART, and IBK, increased to $99.69 \%$, $96.91 \%$ and $99.69 \%$, respectively. Generally, it can be deduced that the classication algorithms, random forest, and IBK are equally suitable for the current data set.

Abdelhamid et al. [11] proposed a multi-agent technology for course recommendation and timetable scheduling system. The academic adviser agent imitates the courseplanning task of the expert academic adviser. It used case data of each student, such as academic level, passed subjects, grades, credit hours, attendance hours of each subject, and prerequisites to recommend the course to students.

Henderson et al. [12] state that the automated webbased advising application system for science and technology faculty at West Indies University to handle general advisory cases and fundamental student issues. Laghari et al. [14] developed a student course planning software package for electrical students to guide course selection process during the registration period. This software guided students to select the most relevant and suitable six courses for the next semester. Laghari [13] presented a system that guides United Arab Emirate University students to choose their semester courses.

Daramola [2] proposed an intelligent course advisory expert system designed and implemented by using rulebased reasoning and case-based reasoning to recommend courses during course registration for a specic semester. The system was based on the academic history of students, and its' performance was evaluated in terms of reliability, usability, and credibility.

Shatnawi et al. [15] state that the application of association rule data mining for selecting and prioritizing courses by generating a list of combinations between different previously registered courses to improve students' performances. This system recommended courses that $t$ to students' status Engin et al. [16] analyzed and discussed about the design, implementation and testing of course advising academic expert system and scholarship suggestion system for undergraduate students for international university.

Al Ahmar [17] stated the development of student advising expert system models to support students' decisions in the selection of course for each academic semester and to help advisers on academic planning. Prescriptive and developmental model, object-oriented database paradigm, and interactive user interface were used in this study.

Hwang [18] proposed the knowledge-based development of an expert system to assist and support students. This system was developed by analyzing the web-based 
problem-solving ability. Two experts evaluated the qualitative and quantitative attributes of the system.

HEls have unique student information systems (SIS) for the requirements or needs of their departments or faculties, including grading system, course ad-vising method as well as rules and regulations. Changing any of these systems and rules cause updates on SIS; therefore, it leads to increase maintenance cost both in terms of financial costs and time.

In this study, we proposed a system that supports advisers to know their students' area of interest and helps to use harmonized knowledge of both students and academicians. It helps to minimize the communication time between advisers and students; thus, they would mainly focus on research and innovative ideas to solve social, economic, and political problems.

Intelligent Personalized Course Advising Model (IPCAM) overcomes the drawbacks of traditional advising systems to use the same structure for all HEls as a framework, which enables flexibly change the rules, regulations, curriculum, and grading system without affecting the persistence of academic data. The development of the model includes the comma-separated values (CSV) file, which provides independence between course recommendation and SIS database. Thus, the compatibility of the system is increased for all HEls and SIS components. The proposal of IPCAM would help to minimize the gaps of HEls and reduce the time spend in course advising.

The rest of the paper is organized as follows: Sects. 2 and 3 present the architecture, and the detailed design and implementation of the proposed system, respectively. Section 4 introduces the evaluation process of the proposed system, and Sect. 5 concludes the remarks of this study.

\section{Architecture of the proposed system}

IPCAM is a specially designed model for the unique needs of each student to provide a suggestion or to help in the decision-making process during the course registration period at the beginning of every semester. The system can easily be merged with the current SIS system and can be integrated without any discrepancy to HEIs SIS. The proposed system is specialized for each student' requirements; thus, students would able to use this system to decide in a fully informed manner without any human advisory or other guides. This leads students to get course suggestions based on their favorite area of interests, their department's curriculum, their academic history, and rules and regulations of the HEI.

\subsection{Data management}

This process ensures the accessibility, reliability, and timeliness of data for students or advice, which includes acquiring, validating, storing, processing, and controlling data. Although data collection has importance, data management has a vital role in software packages.

We have seen different requirements from Wollo University, Cyprus International University, University of Kyrenia, Addis Ababa University, Near East University, Gomel University of Pakistan, King Abdulaziz University of Jeddeh. Direct observation and data analysis had used as the primary data collection method for the first five Universities. Those universities are using student management systems integrated with other systems, but still, they couldn't consist of expert advising system at all. We used only a document analysis method for the rest of the universities $[20,21]$.

According to the collected data, those all HEls are using different SIS portal as per their different requirements including grading system, department's curriculum, course advising method as well as their legislation [20, 21]. They also use different database tools, programming languages, servers, and other technologies. Consistently the change of those requirements leads to expensive SIS maintenance cost.

In this proposed system, a common framework should be considered for data collections that have a direct impact on the effectiveness of the course advising systems such as student, course, instructor attributes, season and academic year. The rules and regulations of HEls, the department's curriculum, and the grading system should also be included in the model for the optimized framework. It is entirely independent of the student information system, which is easy to maintain and reduce system delay time.

All requirements were organized as dependent and independent data. In contrast, dependent data represents academic data, which is typical for all institutions. However, independent data is varied from institute to institute in which they organize and operate in different interacting components of HEI SIS. Figure 1 presents the course attributes from a different perspective.

The transformation mapping method helps to exhibit distinct boundaries between incoming and outgoing data. The course advisory system has critical steps in which to deliver appropriate course advice service to individual students through analyzing academic data from academic datasets which exported or updated from the SIS database or generated from academic experts. Figure 2 shows the summarized block diagram of the integration of academic data storage with the course advisory model. 

data hierarchy
Fig. 1 HEl common academic

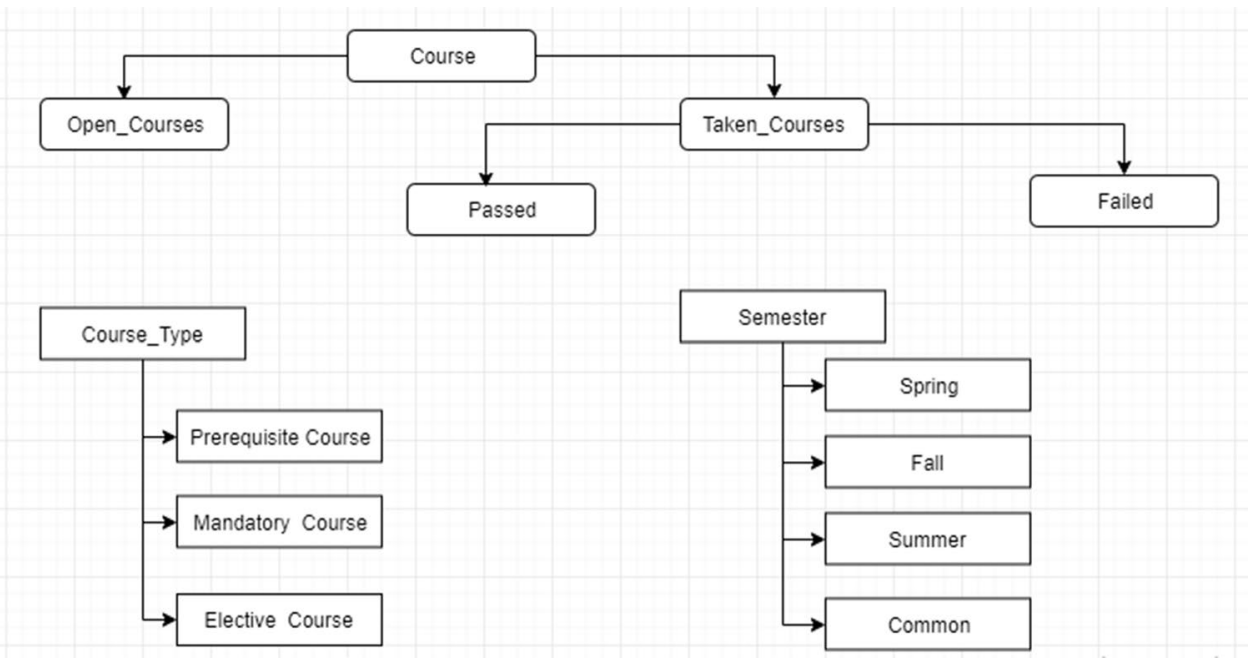

Fig. 2 Academic data storage integration with course advisory model

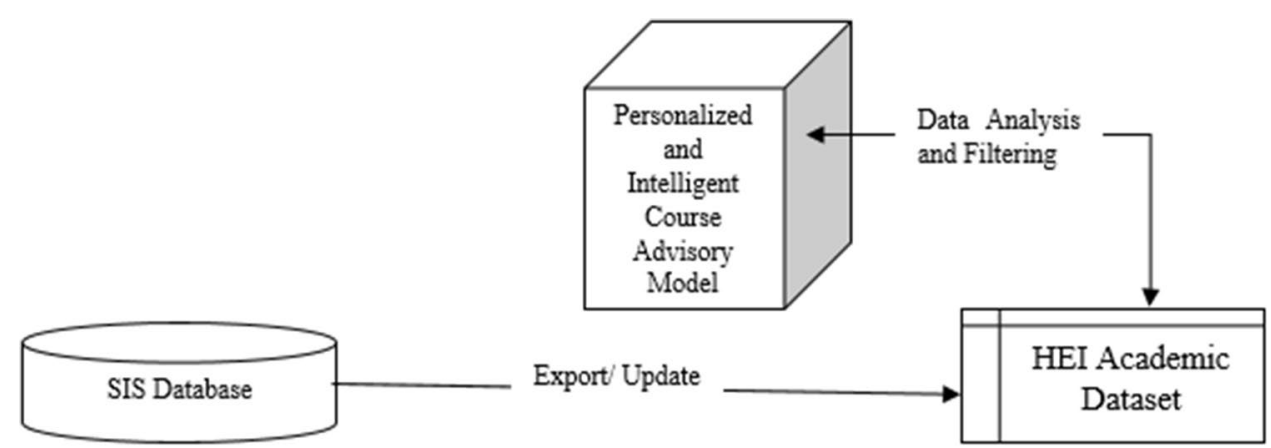

\subsection{Design of the system}

Analyzed requirements were used to transform user needs into some suitable and structured form, which guides the coder in system implementation and maintenance phases.

The architectural design of the academic advisory model is a vital part of maintaining or taking medications to support the developers by presenting the fundamental structure, addressing the key risks, and increasing the reusability and adaptability of the system.

The proposed model is the sub-component of SIS, which can, directly and indirectly, interact with other components of SIS such as SIS authentication, course registration, SIS persistent database, and academic dataset. It automatically gets student data, course, and academic semester information from course registration component. Then, it filters, analyzes, and generates temporary data in the academic dataset. Finally, intelligently suggest courses according to students' academic history and interest.

SIS persistent data storage/database stores the educational information. It retrieves student, course, instructor, and institutional information. It can also generate an academic dataset for the academic advisory model.
Academic dataset store transient data for course advisory model to auto-generate courses for student academic pro le, which filtered from the dataset. It is collective information from the SIS persistent database in which if there is any change in corresponding data of the SIS database, it automatically updates itself.

SIS authentication component gives an interface for students to check their information in SIS persistent database. The student can access any SIS component through this component; therefore, the student authentication result and retrieved student information send to course registration and course advisory components.

The course registration component shows all course and lecturer details from SIS persistent database. All those data then passes to the course advisory model to intelligently filter information. In this component, academician provides vote for field of interest, respective to the course. It provides an interface for course registration for the specific semester in which students register to the particular course after getting advising according to their academic history in the course advisory component. An architectural design of IPCAM integrated HEI SIS components is shown in Fig. 3. 


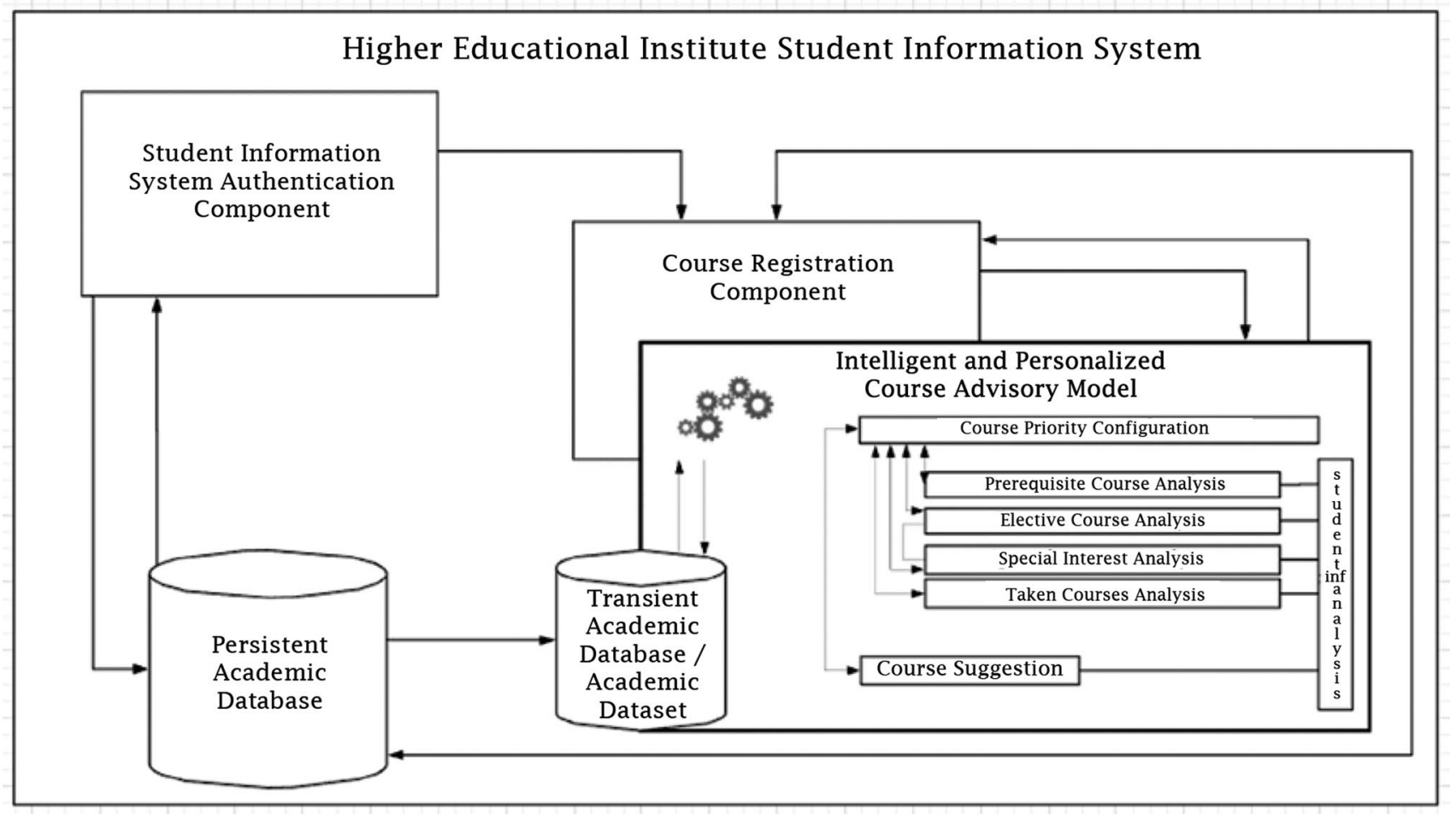

Fig. 3 Architectural design of IPCAM integrated HEI SIS components

\section{Detail design and implementation of IPCAM}

IPCAM has various components in which they collaborate in a structured manner to achieve the ultimate goal of the model. Figure 4 depicts the interaction within the proposed system components, which achieved through receiving and delivering different messages. Student information analysis component provides student's information for taken course analysis, prerequisite course analysis, elective course analysis, individual interest analysis, and course suggestion components. Course, priority configuration component, receive taken courses, prerequisite with their dependent courses, elected courses and favorite courses of each student from taken course analysis, prerequisite course analysis, elective course analysis, and individual course analysis components, respectively. Individual interest analysis components also provide favorite courses to elective course analysis components for further analysis of professionals' ratings. The course suggestion module receives course priority score and student information from course priority configuration and student information analysis, respectively.

Association rule data mining, rule-based expert system, and fundamental recommendation algorithms have been selected and modified for the development of the model based on their suitability for the collected requirements of each component.

Intelligent methods use data mining for the exploration of data patterns. The threshold used for relation is support, confidence, lift, conviction, all confidence, and collective strength. It used for the exploration of frequent patterns, causal structures, associations, or correlations in various datasets from diversified repositories, including transactional and relational data repositories. Association rule data mining is the way to explore patterns in data to find features that occur together and correlate. It mainly involves machine learning for analyzing various data from different patterns or mutual existence in the repository. It determines conditional cooperatives. We had used such a rule in our course dataset where its feature is taken course (1) or not taken (0).

As Table 1 demonstrates, SENG 0031, SENG 0034, SENG 0036, and SENG 0038 were defined as group binary courses under the Mandatory Course set. Enroll $=\left\{\right.$ Trans $_{1}$, Trans $\left._{2}\right\}$ are the group of transactions known as transient database. Every transaction included in the Enroll set contains a subset of courses in the Mandatory Course set. Trans $_{1} \Rightarrow$ Trans $_{2}$, where, Trans 1 is a course or a group of courses. $*$ Trans $_{1}$ : Antecedent, Trans ${ }_{2}$ : Consequent 1 indicates the presence of course in that transaction, and 0 shows the absence. Rule: SENG0031, SENG0034 $\Rightarrow$ $\{$ SENG0038\}, meaning that if the courses SENG0031 and 


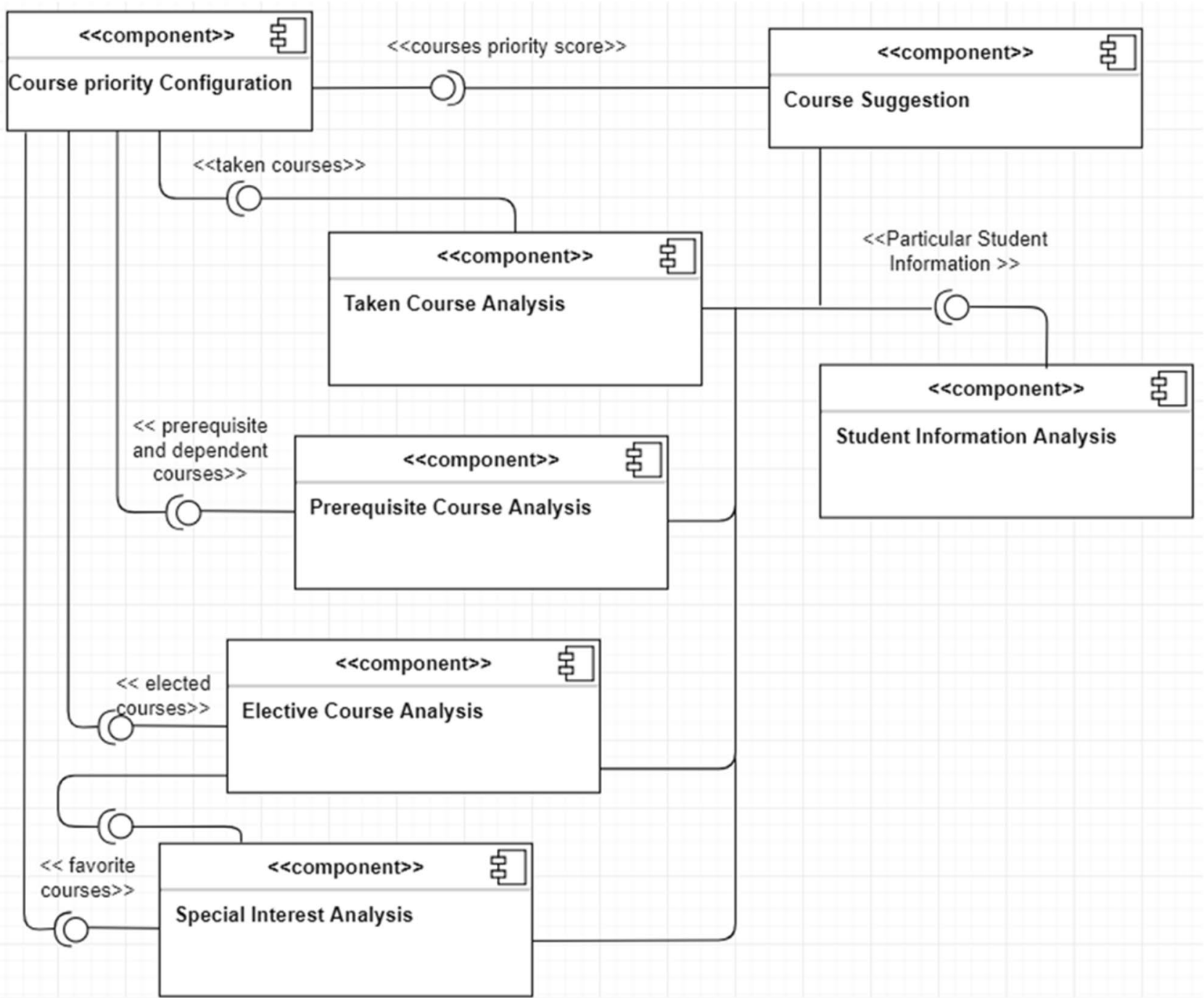

Fig. 4 Functional components integration of IPCAM

Table 1 Mandatory course enrollment transactions

\begin{tabular}{lllll}
\hline $\begin{array}{l}\text { Transac- } \\
\text { tion ID }\end{array}$ & SENG0031 & SENG0034 & SENG0036 & SENG0038 \\
\hline 1 & 1 & 0 & 0 & 0 \\
2 & 1 & 1 & 0 & 1 \\
3 & 1 & 0 & 1 & 0 \\
\hline
\end{tabular}

SENG0034 were taken, the system would send SENG0038 to course priority configuration component for high score value appointment.

Rule-based expert systems belong to what we often called inductive approaches. Inductive approaches will try to induce a "compact" model from the past observations or experiences, and make it generalization. Once this is done, it can forget its experiences and only use its learned model to make a prediction. In those systems, rules represent more knowledge, in which they are if -then - else statements.

The rules considered from the main SISs as institutional rules and departmental rules that govern the overall course priority score value. The conditions under those rules are shown in Fig. 5, which implicitly demonstrates the priority level. Still, the detail priority score value should be adjusted and updated by the SIS administrator from his/her SIS portal, then exported a CSV file. The CSV file is independent from SIS persistent database; however, if any change is happening in the CSV file noting will be changed in the primary system. The course priority configuration components mainly used as source files to create if-then-else rule (course and priority score combination) for different a course that comes from various components of HISs.

\section{SN Applied Sciences}


Fig. 5 Course priority hierarchy and grading policy

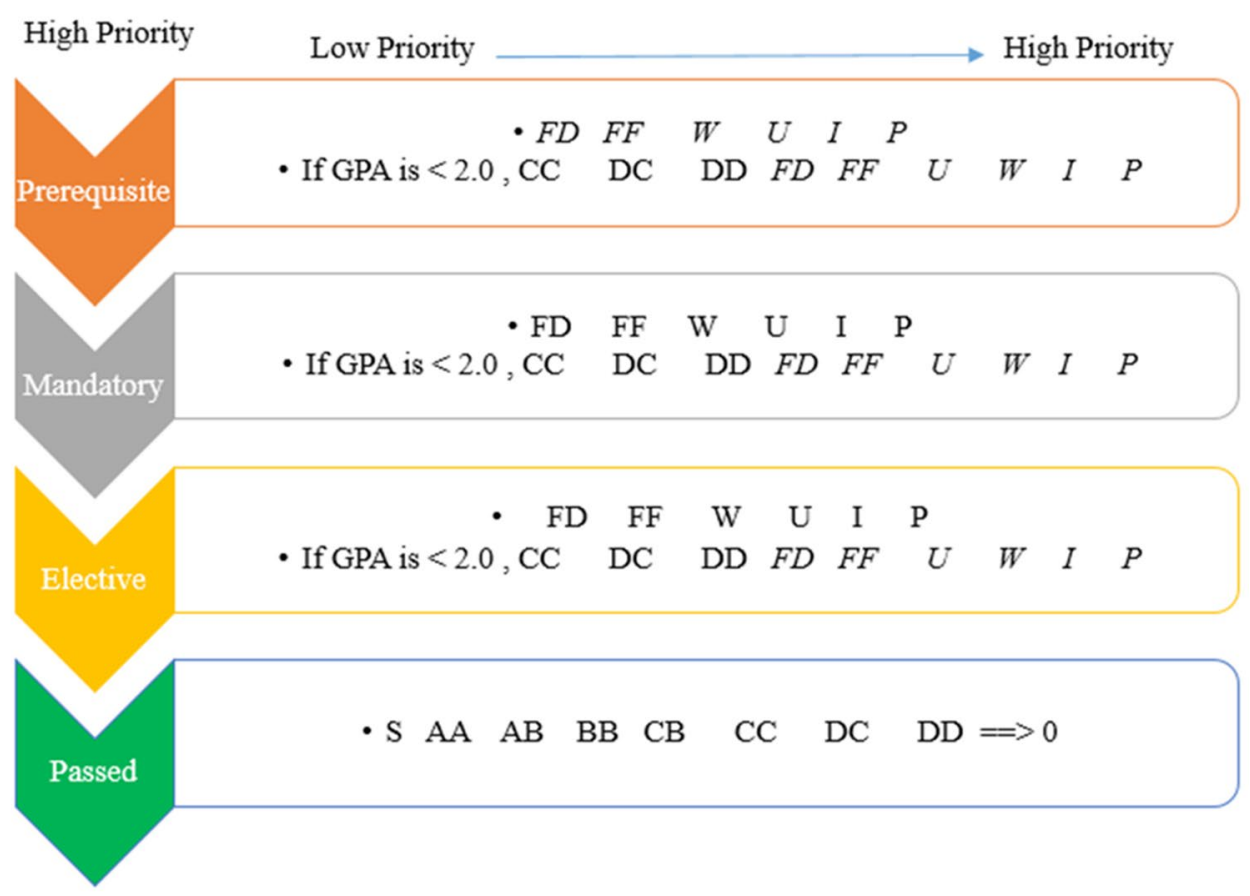

Low Priority

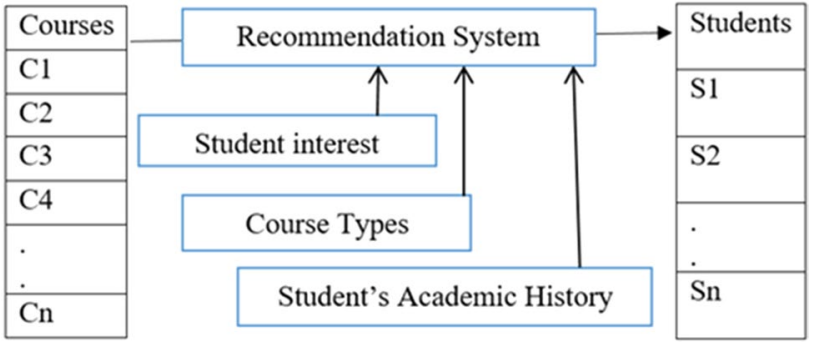

Fig. 6 Simple recommendation for IPCAM

The recommender input relies on the sort of the filtering algorithm used. Votes (Rating) are state expert's views on courses. Usually, those professionals give a rating that obeys rating scale rules, also known as individual scoring ( $1=$ worst, $5=$ best). The collected rating data of experts were organized as a file that has a vital importance for course suggestions. The content of data relies on a semantic assessment of expert's relation with courses.

A suggestion expressed as a roster of $C$ courses where $C$ $\leq n$ expected to be the most appreciated by active pupils. In that case, the usual approach needs this list to include only perceived or rated courses. It is also known as "Top-N recommendation or ranked scoring."

Figure 6 depicts a simple recommendation technique to deliver course suggestions during the beginning of every academic semester as per student's interest, and educational background. Students, experts, and courses are the basic building blocks of IPCAM. Experts giving his or her experiences about many courses and students get a suggestion about elective courses. This technique mainly uses in the course suggestion component to suggest courses through calculating and prioritizing experts rating. In addition to this, the component uses this technique to sort and suggest basic courses according to the course priority value from the course priority configuration component. The ultimate aim of this technique is to generate suggestions for a particular student. The method depends on the input provided, which related to the preferences of those experts.

\subsection{Student information analysis}

It is a fundamental component of the proposed system to personalize course advising through analyzing and determining student-course relation depends on the data that get from the student registration component of HEls SIS. It interacted with other components in different contexts directly or indirectly. It can get the current term and students' batch from the SIS component in a customized manner, which helps to focus on the offered courses and considers taken courses. Algorithm 1 shows the operation of information retrieving and determining the students class level. 


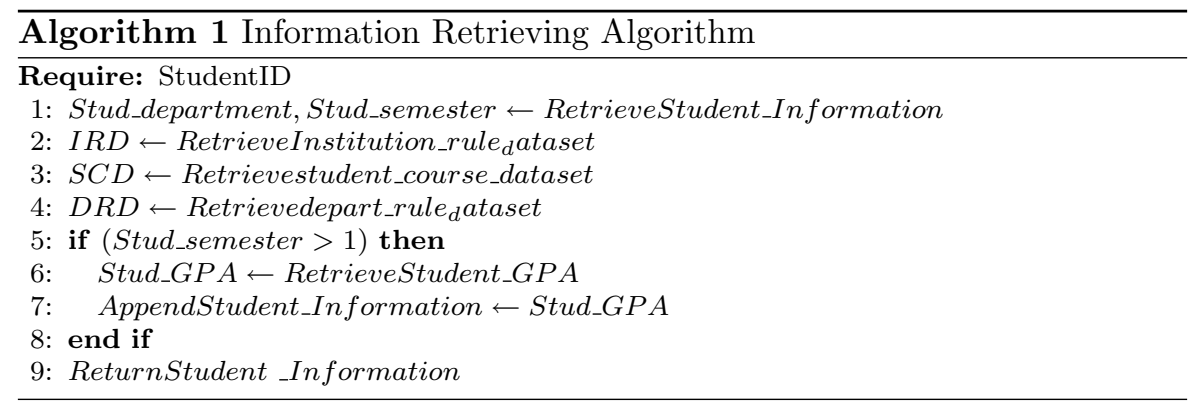

\subsection{Taken course analysis}

It enables to use different student information for filtering and analyzing registered course in the previous semesters. The student has to take a grade for those courses according to his performance, which helps to determine whether the student passed, failed, withdrew, incomplete, satisfied, or unsatisfied courses based on HEl rule set by the system administrator during system configuration. The outcome of this component identifies courses based on course status for a particular student; then, send it to the course priority configuration component for priority scoring. IPCAM algorithms for course analysis of each student can be seen in Algorithm 2.

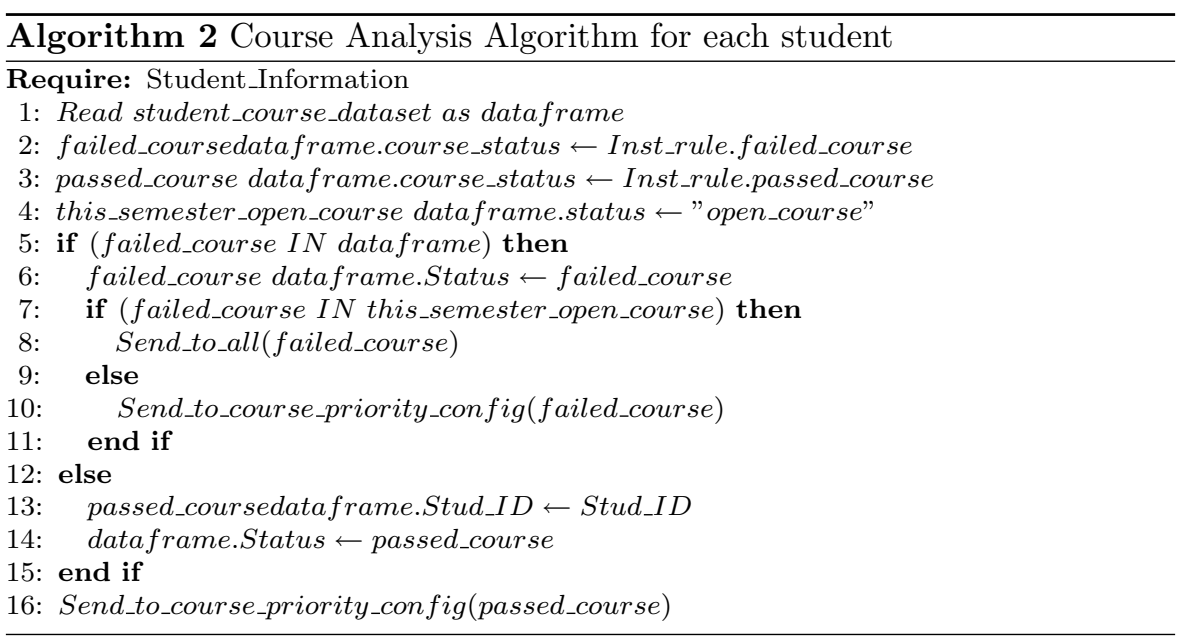




\subsection{Prerequisite analysis}

It is used to identify prerequisites of the ordered courses and to send the course priority configuration to set the priority score. This component primarily focuses on two cases, such as registered (taken) and unregistered courses. If a student failed to pass in prerequisite courses, other dependent courses in the department would send to course priority configuration to get the list priority score. Algorithm 3 presents the procedure for the prerequisite analysis of IPCAM.

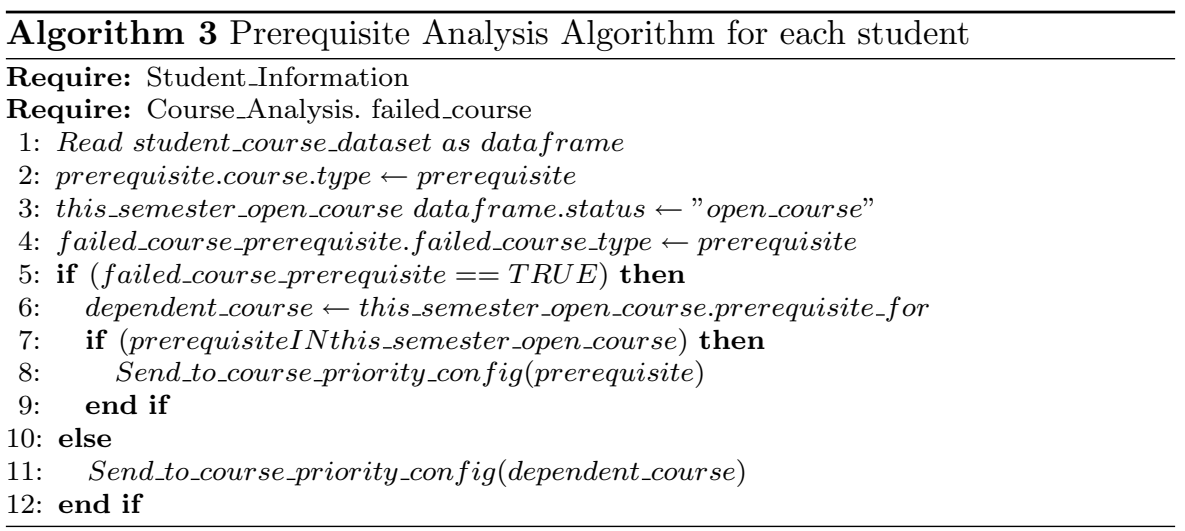

\subsection{Elective course analysis}

It enables filtering and analyzes of all elective courses under their job positions with respective rating in which academic experts gave the score in their portal. Those academic experts shall have the way in SIS to give their vote for course and job position relation according to the current trends of technology, his/her knowledge, skill, and experience. This component requires particular student information, ordered elective courses, and field of specific interest from student information analysis to deliver dependent courses for priority score configuration component as an output. The algorithms of elective course analysis are shown in Algorithm 4 and 5 .

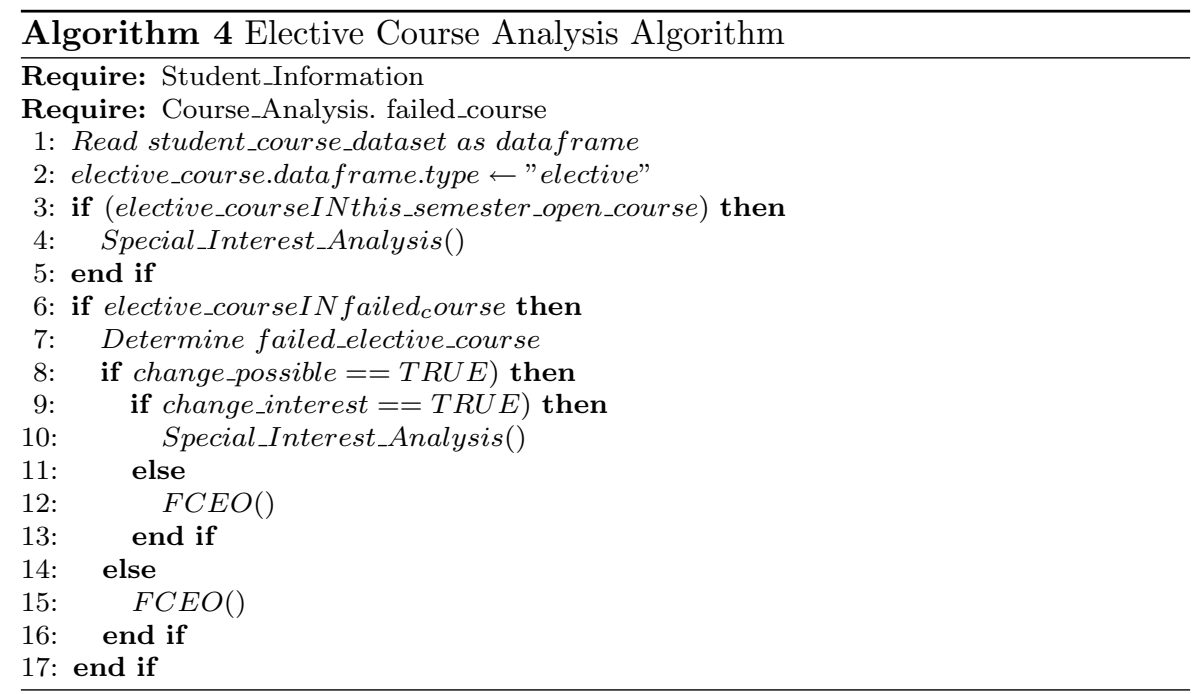




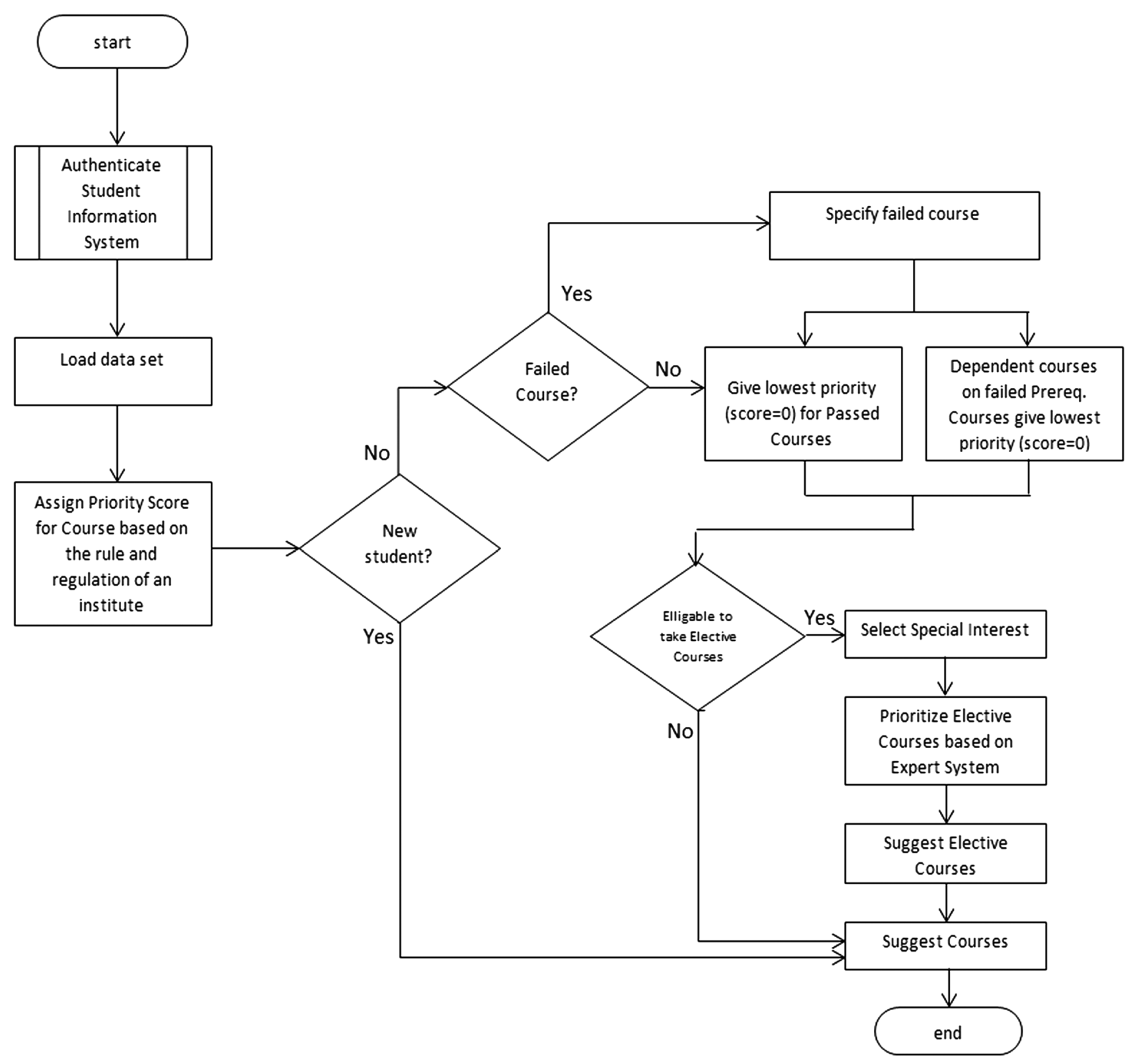

Fig. 7 The flowchart of IPCAM processing

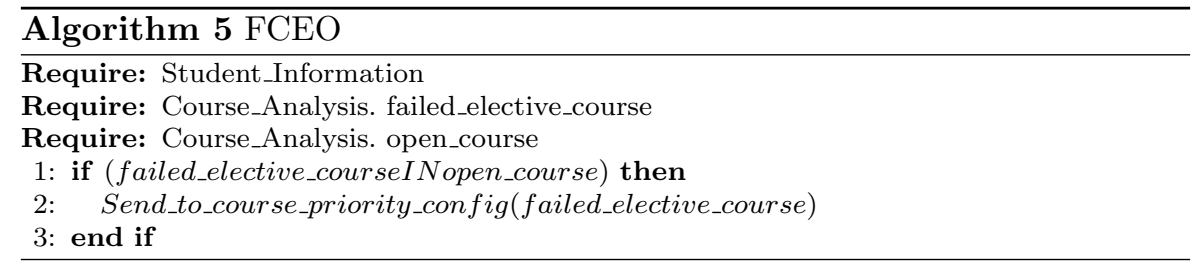

\subsection{Special interest analysis}

It is the smallest component of IPCAM, which receive currently open elective courses from elective course analysis component and students particular interest. Then it delivers altered information to use rating score that collected from academic experts. Algorithm 6 shows the algorithmic principles of special interest analysis of IPCAM. 


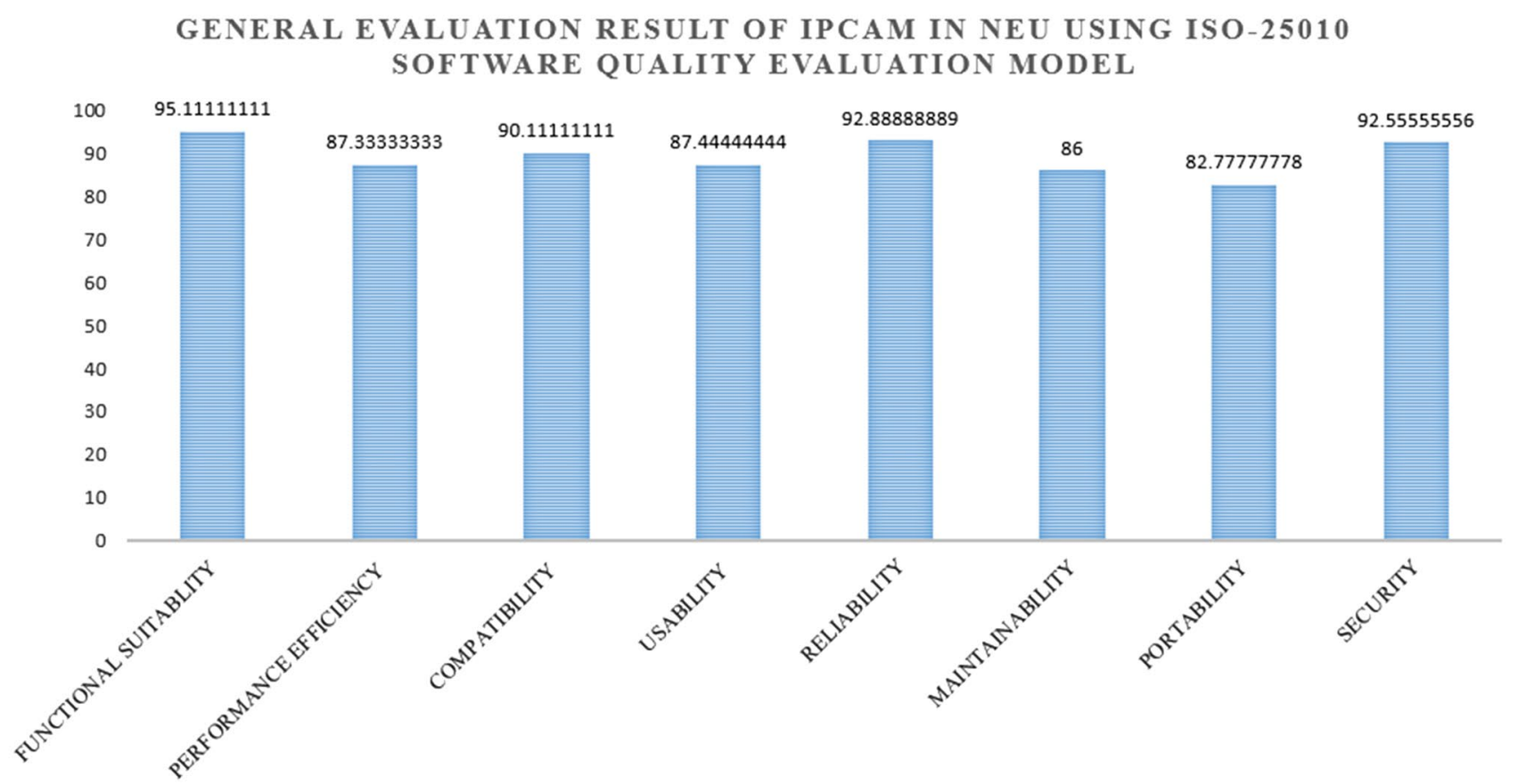

Fig. 8 General evaluation results of the proposed model

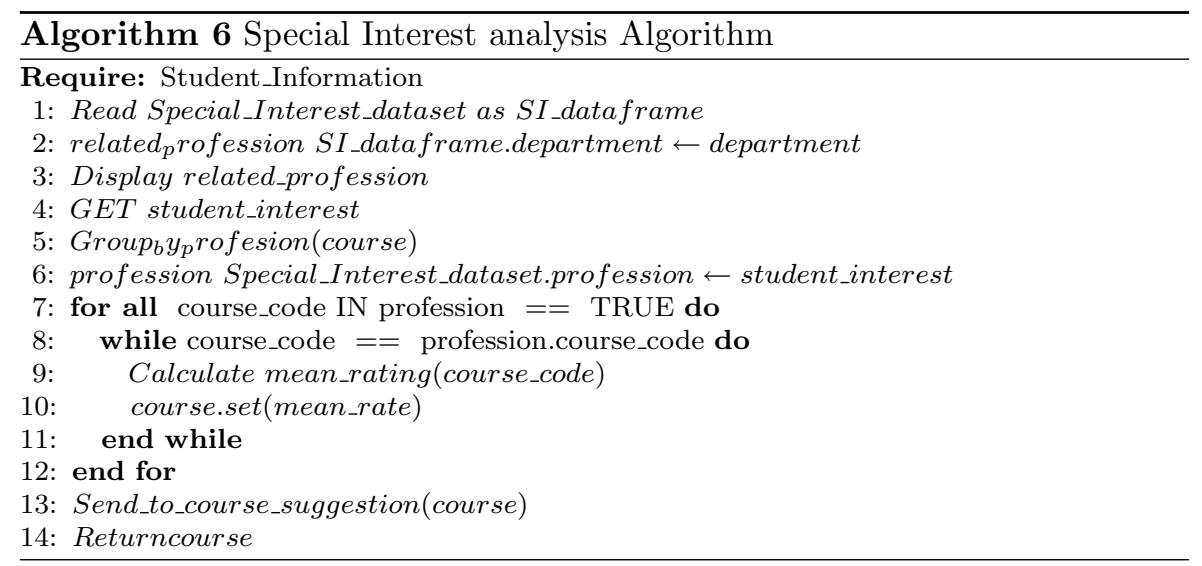

\subsection{Course priority configuration}

It focuses on giving priority orders for courses depend on both the rules and regulations of $\mathrm{HEl}$ and taken/registered courses. Most HEls do not have the same rule and regulations, grading system as well as curriculum. This component compensates their differences through gathering specific and HEI wise information from other SIS components. Therefore, the SIS administrator has the authority to set all the essential information that mentioned above in his SIS portal. This component receives necessary information from SIS to give general priority for prerequisite, mandatory and common courses. Based on the result of other IPCAM components, it sets a priority score for each course. Figure 7 demonstrates the flowchart of IPCAM processing. Algorithm 7 presents the algorithmic details of course priority configuration of IPCAM. 


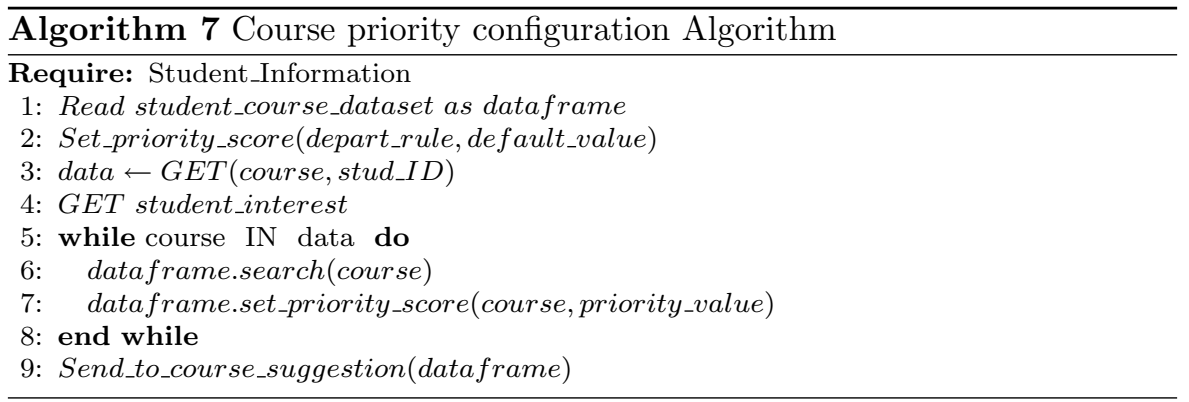

\subsection{Course suggestion}

It comes after the abovementioned series of processes. In this component, two subtasks prioritize and generate a course suggestion for a particular student. Courses are prioritizing through arranging them in order of importance relative to each other. Then according to department plan and curriculum, generate necessary and eligible course suggestions to guide a student to decide on courses.

Information filtering deals with the delivery of information that the students are likely to explore the basic courses depends on their academic history. Academic information filtering system assists students by filtering the data source and deliver necessary information to the students. When the provided information comes in the form of suggestions, the system is called "a recommender system." Due to student's different interests and academic history, this system must filter information in a personalized manner to accommodate the student's interests. Algorithm 8 presents the general principles of course suggestion of IPCAM.

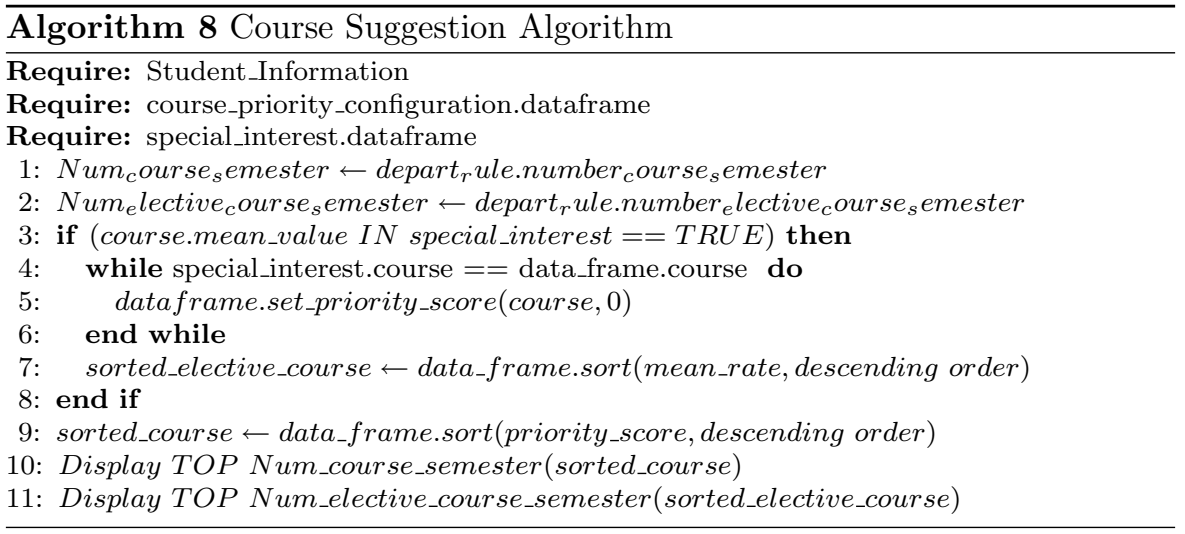

\section{Evaluation of the proposed system}

The most crucial benefit of software evaluation is to gain an understanding of existing or previous software and to help identify future changes. Software evaluation can quickly determine the technical and developmental issues of developed software; besides, it also assesses the general usability and sustainability of software.

Software quality models act as a scheme for software attribute evaluation, which contributes to the quality of the software. They adjust assessing methods or apprises 
a software product. Software designers are planning and developing new applications to meet the exponentially changing requirements.

The proposed IPCAM was evaluated according to the ISO-25010 software evaluation model. ISO-25010 describes a software quality in the quality of product and operating excellence. Operating quality has quality attributes that focus on the output of interaction when a software is active to do in a specific context of use [19]. ISO-25010 is mainly concerned with the quality models definition, which used to clearly explain the desired quality of the software product for software construction and software assessment. The product quality model for software assessment has eight exhaustive quality features. Each characteristic has sets of sub-attributes because of its broad scope.

\subsection{Experimental analysis}

The experiment was performed in the course advising system of the Near East University (NEU), Nicosia, Cyprus, Mersin 10 Turkey, to guide students for selecting suitable courses at the beginning of each semester. IPCAM used the NEU course registration system to take student and course information and then sent recommended courses to the student for his/her approval.

The system requires information from the department head in which they should provide by using their portal. This system also accesses data from the SIS database and student portal as a CSV file and plain text format, respectively.

The proposed system recognizes and recommends courses by comparing the priority level of each course. It consist of courses with priority score. Then, lower priority scores are far to be recommended. The higher priority scores are to be recommended. Prerequisite courses have high priority than mandatory courses, and both have high priority than elective and passed courses. If the previous semester grade point average (GPA) is less than 2.0, the system also suggests courses according to the priority level that stated horizontally.

For those students who passed the previous semester or student's filled courses has not opened in the current semester, the priority order from high to low is as follows prerequisite Mandatory Elective Common courses.

The proposed model was tested by two datasets: namely; General course dataset and the Elective course dataset. The general course dataset is an academic dataset, which consists of three selected departments of the Faculty of Engineering of NEU. These departments were Computer Engineering, Software Engineering, and Information Systems Engineering Departments. The dataset consisted of all offered general courses for these three departments and applied for five students from each batch with their respective classes for the respective semester.

Elective course dataset included elective courses with the rating of their corresponding specialist. Because of the current technology trends, the ratings are not static. Therefore, they have to give a rating score on course profession relation by using their portal on existing SIS to suggest electives courses to achieve students' specialization goals. This dataset consisted of 9 professions, 20 courses, and 1000 rating values.

Fifteen students analyzed the proposed model. The obtained results from the evaluators showed that IPCAM has $87.33 \%$ of performance efficiency, $95.11 \%$ of functional suitability, $87.44 \%$ of usability, $90.11 \%$ of compatibility, $92.89 \%$ of reliability, $82.78 \%$ of portability, $92.56 \%$ of security and $86 \%$ of maintainability. Figure 8 represents the quality features that characterized the overall evaluation result in terms of percentage.

\section{Conclusions}

This article has discussed the analysis and determining problems of an existing academic advising system in various HEls, selecting appropriate course recommendation algorithms, development of highly portable IPCAM, experimental analysis, and model evaluation using the ISO-25010 quality model.

There are some factors, which might affect the result of model evaluation, such as different opinions of evaluators about ISO-25010 quality model, and course ranking in special interest analysis component shall provide by professionals unless it directly affects the result of the recommender. As a future direction, it shall expand the experimental analysis by using more number of departments in various institutes and different evaluation techniques to compare the result with our results and the final result of student's enrolled courses.

\section{Compliance with ethical standards}

Conflict of interest The authors declare that they have no conflict of interest.

\section{References}

1. Concepts of academic advising. https://www.nacada.ksu.edu/ resources/pillars/concept.aspx. Accessed Mar 2019

2. Daramola O, Emebo O, Afolabi I, Ayo C (2014) Implementation of an intelligent course advisory expert system. Int J Adv Res Artif Intell. https://doi.org/10.14569/IJARAI.2014.030502 
3. Marques O, Ding X, Hsu S (2001) Design and development of a web-based academic advising system, pp S3C-6 (2001). https ://doi.org/10.1109/FIE.2001.964053

4. Deorah S, Sridharan S, Goel S (2010) SAES-expert system for advising academic major an Indian perspective. https://doi. org/10.1109/IADCC.2010.5422931

5. Al Nory M (2012) Simple decision support tool for university academic advising, pp 53-57. https://doi.org/10.1109/ITiME .2012 .6291245

6. Alfarsi G, Omar K, Juma M (2017) A rule-based system for advising undergraduate students. J Theor Appl Inf Technol 95:2453-2465

7. Ishak I, Lehat ML (2012) A conceptual framework of web-based academic advisory information system, pp 957-961. https://doi. org/10.1109/SHUSER.2012.6269012

8. Feghali T, Zbib I, Hallal S (2011) A web-based decision support tool for academic advising. Educ Technol Soc 14:82-94

9. Dash P, Vaidhehi V (2017) Enhanced elective subject selection for icse school students using machine learning algorithms. Indian J Sci Technol 10(21):1-10

10. Huang $T$, Zhan $G$, Zhang $H$, Yang $H$ (2017) MCRS: a course recommendation system for moocs. In: Proceedings of The 2017 international conference on advanced technologies enhancing education. ICAT2E 2017

11. Yasser A, Ayoub A, Alhawiti M (2015) Agent-based intelligent academic advisor system. Int J Adv Comput Technol 4(2):1-6

12. Henderson L, Goodridge W (2015) Advise me: an intelligent web-based application for academic advising. Int J Adv Comput Sci Appl 6(8):233-243

13. Laghari M (2014) Automated course advising system. Int J Mach Learn Comput 4(1):47
14. Laghari M, Al Habsi S, Maaz N, Al Naqbi M (2015) A one-semester course planner for EE students. Int J Electron Comput Eng 1(1):13-18

15. Shatnawi R, Qutaibah A, Ghaleb B, Al-Maolegi M (2014) Building a smart academic advising system using association rule mining

16. Engin GEA (2014) Rule-based expert systems for supporting university students. Procedia Comput Sci 31:22-31. https://doi. org/10.1016/j.procs.2014.05.241

17. Ayman M, Ahmar A (2011) A prototype student advising expert system supported with an object-oriented database. Int J Adv Comput Sci Appl. https://doi.org/10.14569/Speciallss ue.2011.010316

18. Hwang G, Chang H (2011) A formative assessment-based mobile learning approach to improving the learning attitudes and achievements of students. Comput Educ 56:1023-1031. https ://doi.org/10.1016/j.compedu.2010.12.002

19. Miguel J, Mauricio D, Rodriguez G (2014) A review of software quality models for the evaluation of software products. Int J Softw Eng Appl 5:31-54. https://doi.org/10.5121/ijsea .2014 .5603

20. Al-Ghamdi A et al (2012) An expert system for advising postgraduate students. Int J Comput Sci Inf Technol IJCSIT 3:4529-4532

21. Aslam MZ, Nasimullah, Khan AR (2011) A proposed decision support system/expert system for guiding fresh students in selecting a faculty in Gomal University, Pakistan. arXiv preprint arXiv :1104.1678

Publisher's Note Springer Nature remains neutral with regard to jurisdictional claims in published maps and institutional affiliations. 